Journal of Optimization Theory and Applications: Vol. 114, No.1 pp.27-54, July 2002.

\title{
Relaxation through Homogenization for Optimal Design Problems with Gradient Constraints ${ }^{1}$
}

\author{
R. LIPTON \\ Department of Mathematics \\ LOUisiana State University \\ Baton Rouge, Louisiana 70803 \\ lipton@math.lsu.edu
}

\begin{abstract}
The problem of relaxation of optimal design problems for multiphase composite structures in the presence of constraints on the gradient of the state variable is addressed. A relaxed formulation for the problem is given in the presence of a finite or infinite number of constraints. The relaxed formulation is used to identify minimizing sequences of configurations of phases.
\end{abstract}

Key Words. Gradient constraints, relaxation, optimal design.

\footnotetext{
${ }^{1}$ This work was supported by the NSF, Grant DMS-0072469 and by the Air Force Office of Scientific Research, Grant F49620-99-1-0009.
} 


\section{Introduction}

In the study of optimal design of composite structures, much effort has focused on problems of optimization of design criteria in the absence of constraints on the gradients of field variables. In this article a theory is developed for the relaxation of optimal design problems for multiphase composite structures in the presence of constraints on the gradient. An example of where such a theory would be useful is the design of a multi-phase insulator with a prescribed overall dielectric constant subject to the constraint that inside the composite the electric field does not exceed a critical value. This type of problem is important in the applications (Ref. 1), where large values of the local electric field are attributed to material breakdown (Ref. 2).

To fix ideas the paper is written in the physical context of multiphase dielectric materials. The design domain is a bounded open set $\Omega$ in $R^{m}$ and the multi-phase dielectric is made up of $N$ anisotropic dielectric materials with dielectric constants given by the $m \times m$ symmetric positive matrices $A_{1}, A_{2}, A_{3}, \ldots, A_{N}$. A particular choice of component dielectric constants is specified by the array $\underline{A}=\left(A_{1}, A_{2}, A_{3}, \ldots, A_{N}\right)$ and the dielectric constant at each point in the design domain is given by $C(\underline{A}, x)=\sum_{i=1}^{N} \chi_{i}(x) A_{i}$. Here $\chi_{i}$ is the indicator function of the set occupied by the $i^{\text {th }}$ dielectric phase and $\chi_{i}=1$ for $x$ in the $i^{\text {th }}$ phase and zero elsewhere. The design space consists of all partitions of $\Omega$ into $N$ Lebesgue measurable subsets $\Omega_{1}, \Omega_{2}, \ldots, \Omega_{N}$ occupied by the different dielectrics subject to the resource constraints meas $\left(\Omega_{i}\right) \leq \gamma_{i}$. Here $\sum_{i} \gamma_{i} \geq$ meas $(\Omega)$ and the vector of resource constraints is written as $\underline{\gamma}=\left(\gamma_{1}, \gamma_{2}, \ldots, \gamma_{N}\right)$. The electric potential in the composite is denoted by $\phi$ and for a prescribed distributed charge density $f$ satisfies

$$
-\operatorname{div}(C(\underline{A}, x) \nabla \phi)=f .
$$

Here the potential $\phi$ is zero on the boundary of $\Omega$. From the theory of Poisson's equation it is well known that the potential lies in the Sobolev space $H_{0}^{1}(\Omega)$ and $f$ can be chosen from the dual space $H^{-1}(\Omega)$. The design criteria is a function of the potential and is denoted by $F(\phi)$. The type of criteria considered here are those associated with overall energy dissipation given by $F(\phi)=\int_{\Omega} \phi f d x$ or the distance of the potential from a desired target potential $\hat{\phi}$ given by $F(\phi)=\int_{\Omega}|\phi-\hat{\phi}|^{2} d x$. Both of these objective functions are continuous with respect to weak convergence in $H_{0}^{1}(\Omega)$. The results given here are not restricted to this situation and apply to any design criteria that is continuous with respect to $G$ convergent sequences of designs (Ref. 3).

For a given tolerance $K>0$ and a prescribed nonnegative function $p(x)$ the basic gradient constraint is given by

$$
\int_{\Omega} p(x)|\nabla \phi|^{2} d x \leq K^{2} .
$$

For simplicity $p(x)$ is chosen to be in the space of infinitely differentiable functions continuous on the closure of $\Omega$, i.e., $p(x)$ in $C^{\infty}(\bar{\Omega})$. For a finite collection of functions $p_{1}, p_{2}, \ldots, p_{j}$ one has the set of constraints given by

$$
\int_{\Omega} p_{k}(x)|\nabla \phi|^{2} d x \leq K^{2}, p_{k}(x) \geq 0, k=1,2, \ldots, j .
$$


Last one may consider the infinite number of constraints given by

$$
\int_{\Omega} p(x)|\nabla \phi|^{2} d x \leq K^{2} \int_{\Omega} p(x) d x, \text { for all } p(x) \geq 0 \text { in } C^{\infty}(\bar{\Omega}),
$$

which is equivalent to the local constraint

$$
|\nabla \phi| \leq K, \text { almost everywhere in } \Omega
$$

The basic gradient constrained optimal design problem is given by

$$
P=\inf _{\substack{\text { Configurations, } \\ \text { meas }\left(\Omega_{i}\right) \leq \gamma_{i}}} F(\phi)
$$

where $\phi$ is a solution of the equation of state (1) and is subject to the gradient constraint given by (2). Optimal design problems subject to a finite or an infinite number of gradient constraints are formulated in the same way.

The goal of this analysis is to present a theory of relaxation for these problems that provides the theoretical underpinnings for a numerical approach to the solution of the design problem. The problem as stated above is not readily amenable to numerical solution. The fundamental reason for this is that problems of this type do not possess configurations for which the infimum in (6) is obtained, see (Refs. 4-6). Thus any approach that seeks to identify optimal configurations is most likely to fail. Instead one must seek a methodology for systematically identifying minimizing sequences of configurations that approach the infimum of (6).

In the absence of gradient constraints, problems of this kind have been the object of intense and fruitful study. One approach to these problems is the relaxation of the design problem through the extension of the design space. This is accomplished using the theory of homogenization. This approach can be found in the seminal work of (Ref. 7) and (Ref. 8). The approach taken here follows the philosophy given in (Ref. 7) and (Ref. 8) and provides a relaxation of the design problem (6) through an extension of the design space. However the extended design space for the gradient constrained design problem is seen to be a generalization of the one developed for the unconstrained problem. Loosely speaking, in the absence of gradient constraints the design space is extended to include all effective dielectric tensors made from composites formed from the constituent dielectric materials. However for gradient constrained optimal design problems it is found that the relaxed problem requires the extension of the design space to include all effective dielectric tensors viewed as functions of the dielectric tensors of the constituent materials.

In order to proceed the optimal design problem over configurations is reformulated in an equivalent way. For a given array $\underline{A}$ a neighborhood $\mathcal{N}(\underline{A})$ of $\underline{A}$ is introduced. Arrays in this neighborhood are denoted by $\underline{P}=\left(P_{1}, P_{2}, \ldots, P_{N}\right)$. The neighborhood is chosen such that all matrices $P_{i}$ in the array $\underline{P}$ satisfy the constraint $0<\lambda<P_{i}<\Lambda$. The associated set of controls $C(\underline{P}, x)=\sum_{i=1}^{N} \chi_{i}(x) P_{i}$ for $\underline{P}$ in the neighborhood $\mathcal{N}(\underline{A})$ for which $\int_{\Omega} \chi_{i} d x \leq \gamma_{i}$ is denoted by $\mathcal{C}(\underline{A}, \underline{\gamma})$ and the design problem is given by

$$
P=\inf _{C(\underline{P}, x)} \operatorname{in} \mathcal{C}(\underline{A}, \underline{\gamma})
$$


where $\phi$ is a solution of the equation of state (1) and is subject to the gradient constraint given by (2). As before optimal design problems subject to a finite or an infinite number of gradient constraints are formulated in the same way.

The notion of homogenization is given in the theories of $\mathrm{G}$ convergence and $\mathrm{H}$ convergence, see (Ref. 9) and (Ref. 10). Both notions of convergence coincide for the problem addressed here. A comprehensive introduction to the theory of $\mathrm{G}$ convergence together with extensive references to the literature is given in (Ref. 11).

Definition 1.1 G Convergence. The sequence $\left\{C^{n}(\underline{P}, x)\right\}_{n=1}^{\infty} \mathrm{G}$ converges to $C^{\infty}(\underline{P}, x)$ if and only if, for any open set $\omega \subset \subset \Omega$ and any $f$ in $H^{-1}(\omega)$, the solutions $w^{n}$ in $H_{0}^{1}(\omega)$ of

$$
-\operatorname{div}\left(C^{n}(\underline{P}, x) \nabla w^{n}\right)=f
$$

satisfy $w^{n} \rightarrow w$ weakly in $H_{0}^{1}(\omega), C^{n}(\underline{P}, x) \nabla w^{n} \rightarrow C^{\infty}(\underline{P}, x) \nabla w$ weakly in $L^{2}(\omega)^{N}$, where $w$ is the $H_{0}^{1}(\omega)$ solution of

$$
-\operatorname{div}\left(C^{\infty}(\underline{P}, x) \nabla w\right)=f .
$$

The resource constraints for a G convergent sequence of controls is handled in the usual way by assuming that the associated sequence of characteristic functions $\left\{\chi_{i}^{n}\right\}_{n=1}^{\infty}$ satisfy

$$
\chi_{i}^{n} \rightarrow \theta_{i} \text { in } L^{\infty}(\Omega) \text { weak } * .
$$

The design space is extended to include the set of all functions $C^{\infty}(\underline{P}, x)$ for which there exists a sequence of controls $\left\{C^{n}(\underline{P}, x)\right\}_{n=1}^{\infty} \mathrm{G}$ converging to $C^{\infty}(\underline{P}, x)$ for all $\underline{P}$ in $\mathcal{N}(\underline{A})$ and $\int_{\Omega} \theta_{i} d x \leq \gamma_{i}$. The set of all such functions $C^{\infty}(\underline{P}, x)$ is the G-closure of the set $\mathcal{C}(\underline{A}, \underline{\gamma})$ and is denoted by $\mathcal{G C}(\underline{A}, \gamma)$. We point out that $\mathcal{C}(\underline{A}, \gamma)$ is contained in $\mathcal{G C}(\underline{A}, \gamma)$. Its evident that the extension of design space introduced here is more general than the usual one in that it involves the notion of tensor valued effective dielectric functions as opposed to effective dielectric tensors.

The next step in the relaxation procedure is the correct description of the gradient constraints $(2-5)$ in the extended design space. Given a sequence $\left\{C^{n}(\underline{P}, x)\right\}_{n=1}^{\infty}$ that $\mathrm{G}$ converges to $C^{\infty}(\underline{P}, x)$ for every $\underline{P}$ in $\mathcal{N}(\underline{A})$ the local corrector function associated with the sequence $\left\{C^{n}(\underline{A}, x)\right\}_{n=1}^{\infty}$ is introduced. Consider a cube $Q(x, r)$ of sidelength $r$ centered at some point $x$ in $\Omega$. For $r$ sufficiently small $Q(x, r)$ is contained within $\Omega$. Given a vector $E$ in $R^{N}$, the local corrector function is written as $\varphi_{E}^{n, r}$ where $\varphi_{E}^{n, r}$ is the $H_{0}^{1}(Q(x, r))$ solution of

$$
-\operatorname{div}\left(C^{n}(\underline{A}, y)\left(\nabla \varphi_{E}^{n, r}+E\right)\right)=-\operatorname{div}\left(C^{\infty}(\underline{A}, y) E\right) \text {, for } y \text { in } Q(x, r) .
$$

The local corrector functions are used to define directional derivatives of the G-limit $C^{\infty}(\underline{P}, x)$ in the following theorem.

Theorem 1.1 Given that $\left\{C^{n}(\underline{P}, x)\right\}_{n=1}^{\infty}$ G converges to $C^{\infty}(\underline{P}, x)$ for every $\underline{P}$ in $\mathcal{N}(\underline{A})$, then the directional derivative of $C^{\infty}(\underline{P}, x)$ at $\underline{A}$ with respect to the $i^{\text {th }}$ component conductivity in the direction specified by the $m \times m$ symmetric matrix $M_{i}$ is given by

$$
\frac{\partial C^{\infty}}{\partial M_{i}} E \cdot E=\sum_{k=1}^{m} \sum_{l=1}^{m}\left[M_{i}\right]_{k l}\left(\lim _{r \rightarrow 0} \lim _{n \rightarrow \infty}(1 /|Q(x, r)|) \int_{Q(x, r)} \chi_{i}^{n}\left(\nabla_{k} \varphi_{E}^{n, r}+E_{k}\right)\left(\nabla_{l} \varphi_{E}^{n, r}+E_{l}\right) d y\right)
$$

and exists almost everywhere in $\Omega$ and for every $E$ in $R^{m}$. 
From Theorem 1.1 it follows that the definition of the $i^{\text {th }}$ phase gradient denoted by $\left(\nabla_{k l}^{i} C^{\infty}(\underline{A}, x)\right) E \cdot E$ is given by the local formula

$$
\left(\nabla_{k l}^{i} C^{\infty}(\underline{A}, x)\right) E \cdot E=\lim _{r \rightarrow 0} \lim _{n \rightarrow \infty}(1 /|Q(x, r)|) \int_{Q(x, r)} \chi_{i}^{n}\left(\nabla_{k} \varphi_{E}^{n, r}+E_{k}\right)\left(\nabla_{l} \varphi_{E}^{n, r}+E_{l}\right) d y
$$

and

$$
\left.\left(\nabla_{k k}^{i} C^{\infty}(\underline{A}, x)\right) E \cdot E=\lim _{r \rightarrow 0} \lim _{n \rightarrow \infty}(1 /|Q(x, r)|) \int_{Q(x, r)} \chi_{i}^{n} \mid \nabla \varphi_{E}^{n, r}+E\right)\left.\right|^{2} d y
$$

where repeated indicies indicate summation. Theorem 1.1 provides explicit formulas for the derivatives of the G-limit in terms of the microscopic problems (11). The identification of the suitable microscopic problems together with the local formulas (12-14) are necessary for numerical solution schemes based on relaxation through homogenization, see (Ref. 12). General differentiability properties of G-limits obtained without the use of local formulas are presented in (Ref. 13). Differentiability properties for the the effective dielectric tensor in the contexts of periodic homogenization and statistically homogeneous random media were presented in (Ref. 14).

The relaxation scheme developed here follows from the homogenization result given below. In order to state the result one considers a sequence of configurations and a function $C^{\infty}(\underline{P}, x)$ for which the sequence of controls $\left\{C^{n}(\underline{P}, x)\right\}_{n=1}^{\infty}$ G converges to $C^{\infty}(\underline{P}, x)$ for every $\underline{P}$ in $\mathcal{N}(\underline{A})$. From homogenization theory there exists a potential $\phi^{\infty}$ such that the sequence of potentials $\left\{\phi^{n}\right\}_{n=1}^{\infty}$ in $H_{0}^{1}(\Omega)$ satisfying

$$
-\operatorname{div}\left(C^{n}(\underline{A}, x) \nabla \phi^{n}\right)=f
$$

converges weakly to $\phi^{\infty}$ in $H_{0}^{1}(\Omega)$ where

$$
-\operatorname{div}\left(C^{\infty}(\underline{A}, x) \nabla \phi^{\infty}\right)=f .
$$

The following homogenization theorem allows one to pass to the homogenization limit in the gradient constraints of the form $(2-5)$.

Theorem 1.2 The Homogenization Limit of Weakly Converging Gradients.

For any function $p(x)$ in $C^{\infty}(\bar{\Omega})$

$$
\lim _{n \rightarrow \infty} \int_{\Omega} p(x)\left|\nabla \phi^{n}\right|^{2} d x=\int_{\Omega} p(x)(\sigma(x)+I) \nabla \phi^{\infty} \cdot \nabla \phi^{\infty} d x,
$$

where the covariance matrix $\sigma(x)$ is a non negative matrix defined by

$$
\sigma(x) E \cdot E=\sum_{i=1}^{N}\left(\nabla_{k k}^{i} C^{\infty}(\underline{A}, x)\right) E \cdot E-|E|^{2},
$$

for all vectors $E$ in $R^{m}$.

The homogenization limit of the gradient constraints is given in the following corollary 
Corollary 1.1 The Homogenization Limit of Gradient Constraints.

If for some nonnegative $p$ in $C^{\infty}(\bar{\Omega})$ it is known that

$$
\int_{\Omega} p(x)\left|\nabla \phi^{n}\right|^{2} d x \leq K^{2}
$$

for the sequence $\left\{\phi^{n}\right\}_{n=1}^{\infty}$ given above, then $\nabla \phi^{\infty}$ satisfies

$$
\int_{\Omega} p(x)(\sigma(x)+I) \nabla \phi^{\infty} \cdot \nabla \phi^{\infty} d x \leq K^{2} .
$$

Similarly if one has the local constraint $\left|\nabla \phi^{n}\right| \leq K$ almost everywhere in $\Omega$ then $\nabla \phi^{\infty}$ satisfies

$$
\sqrt{(\sigma(x)+I) \nabla \phi^{\infty} \cdot \nabla \phi^{\infty}} \leq K
$$

almost everywhere in $\Omega$.

The covariance can be thought of as a new effective property characterizing the interaction between the local microstructure and the macroscopic electric field $-\nabla \phi^{\infty}$. It has been introduced in the contexts of periodic and statistically homogeneous random composites in (Refs. 15, 16). In the general context of $\mathrm{G}$ convergent sequences of matrices the local formula for $\sigma$ is given in Theorem 1.1 of Ref. 17 .

The relaxed formulation for the basic gradient constrained optimal design problem is introduced. For $p(x) \geq 0$ and $p(x)$ in $C^{\infty}(\bar{\Omega})$, the relaxed formulation of $(7)$ is

$$
Q P=\inf _{C^{\infty}(\underline{P}, x) \operatorname{in} \mathcal{G C}(\underline{A}, \underline{\gamma})} F(\phi),
$$

subject to the constraint:

$$
\int_{\Omega} p(x)(\sigma(x)+I) \nabla \phi \cdot \nabla \phi d x \leq K^{2}
$$

where $\phi$ is the $H_{0}^{1}(\Omega)$ solution to the state equation

$$
-\operatorname{div}\left(C^{\infty}(\underline{A}, x) \nabla \phi\right)=f,
$$

and $\sigma$ is defined by (18).

The first important feature of the relaxed problem is that the infimum of the relaxed design problem is attained by a control in $\mathcal{G C}(\underline{A}, \underline{\gamma})$, i.e.,

Theorem 1.3 There exists a control $\hat{C}^{\infty}(\underline{P}, x)$ in $\mathcal{G C}(\underline{A}, \underline{\gamma})$ and state variable $\hat{\phi}$ in $H_{0}^{1}(\Omega)$ for which

$$
-\operatorname{div}\left(\hat{C}^{\infty}(\underline{A}, x) \nabla \hat{\phi}\right)=f
$$

and

$$
Q P=F(\hat{\phi})
$$

where

$$
\int_{\Omega} p(x)(\hat{\sigma}(x)+I) \nabla \hat{\phi} \cdot \nabla \hat{\phi} d x \leq K^{2}
$$

and

$$
\hat{\sigma}(x) E \cdot E=\sum_{i=1}^{N}\left(\nabla_{k k}^{i} \hat{C}^{\infty}(\underline{A}, x)\right) E \cdot E-|E|^{2},
$$

for every $E$ in $R^{m}$. 
The second important feature is the connection between the minimizer $\hat{C}^{\infty}(\underline{P}, x)$ of the relaxed problem and minimizing sequences of configurations. To make the connection the following optimal design problems are introduced. For $p(x) \geq 0$ and $p(x)$ in $C^{\infty}(\bar{\Omega})$,

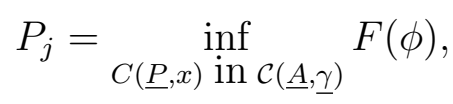

subject to the constraint:

$$
\int_{\Omega} p(x)|\nabla \phi|^{2} d x \leq K^{2}(1+1 / j)
$$

where $\phi$ is the $H_{0}^{1}(\Omega)$ solution to the state equation

$$
-\operatorname{div}(C(\underline{A}, x) \nabla \phi)=f
$$

Its clear that as $j$ tends to infinity the constraints given in the design problems $P_{j}$ approach the constraint associated with (7). The problems $P_{j}$ share the same feature as $(7)$ in that they are optimal design problems over configurations of $N$ dielectrics. The connection between the minimizer of the relaxed problem and minimizing sequences of configurations is given in the following theorem.

Theorem 1.4 Given a minimizer $\hat{C}^{\infty}(\underline{P}, x)$ of $Q P$ then there is a sequence of configurations and associated controls $\left\{C^{j}(\underline{P}, x)\right\}_{j=1}^{\infty}$ in $\mathcal{C}(\underline{A}, \underline{\gamma})$ such that for all $\underline{P}$ in $\mathcal{N}(\underline{A})$, the sequence $\left\{C^{j}(\underline{P}, x)\right\}_{j=1}^{\infty}$ G-converges to $\hat{C}^{\infty}(\underline{P}, x)$ and the state variables $\phi^{j}$ satisfy the constraints (30). For this case one has

$$
\lim _{j \rightarrow \infty} F\left(\phi^{j}\right)=Q P
$$

Moreover, given any $\epsilon>0$ there exists an index $J>0$ such that for all $j>J$,

$$
P_{j} \leq F\left(\phi^{j}\right) \leq P_{j}+\epsilon
$$

and

$$
\lim _{j \rightarrow \infty} P_{j}=Q P
$$

Thus one can use the minimizer for the relaxed problem to recover nearly optimal constrained gradient designs of $N$ dielectrics.

The locally constrained optimal design problem is given by

$$
\inf _{C(\underline{P}, x) \text { in } \mathcal{C}(\underline{A}, \underline{\gamma})} F(\phi)
$$

where $\phi$ is a $H_{0}^{1}(\Omega)$ solution of the equation of state (1) and is subject to the gradient constraint given by

$$
|\nabla \phi| \leq K, \text { almost everywhere in } \Omega \text {. }
$$


The local constraint can also be given in the equivalent form (4). Moreover choosing a countable dense subset $\left\{p_{k}\right\}_{k=1}^{\infty}$ of the set of nonnegative functions in $C^{\infty}(\bar{\Omega})$ the local constraint is equivalent to

$$
\int_{\Omega} p_{k}(x)|\nabla \phi|^{2} d x \leq K^{2} \int_{\Omega} p_{k}(x) d x, \text { for } 1 \leq k<\infty .
$$

The relaxed formulation of $(35)$ is

$$
Q R=\inf _{C^{\infty}(\underline{P}, x) \text { in } \mathcal{G C}(\underline{A}, \underline{\gamma})} F(\phi),
$$

subject to the local constraint:

$$
\sqrt{(\sigma(x)+I) \nabla \phi \cdot \nabla \phi} \leq K \text { almost everywhere in } \Omega
$$

where $\phi$ is the $H_{0}^{1}(\Omega)$ solution to the state equation

$$
-\operatorname{div}\left(C^{\infty}(\underline{A}, x) \nabla \phi\right)=f,
$$

and $\sigma$ is defined by (18). The first important feature of the relaxed problem is that the infimum of the design problem is attained by a control in $\mathcal{G C}(\underline{A}, \underline{\gamma})$, i.e.,

Theorem 1.5 There exists a control $\hat{C}^{\infty}(\underline{P}, x)$ in $\mathcal{G C}(\underline{A}, \underline{\gamma})$ and state variable $\hat{\phi}$ in $H_{0}^{1}(\Omega)$ for which

$$
-\operatorname{div}\left(\hat{C}^{\infty}(\underline{A}, x) \nabla \hat{\phi}\right)=f
$$

and

$$
Q R=F(\hat{\phi})
$$

where

$$
\sqrt{(\hat{\sigma}(x)+I) \nabla \hat{\phi} \cdot \nabla \hat{\phi}} \leq K \text { almost everywhere in } \Omega
$$

and

$$
\hat{\sigma}(x) E \cdot E=\sum_{i=1}^{N}\left(\nabla_{k k}^{i} \hat{C}^{\infty}(\underline{A}, x)\right) E \cdot E-|E|^{2}
$$

for every $E$ in $R^{m}$.

The second important feature is the connection between the minimizer $\hat{C}^{\infty}(\underline{P}, x)$ of the relaxed problem and minimizing sequences of configurations. To make the connection consider a countable dense subset $\left\{p_{k}\right\}_{k=1}^{\infty}$ of the nonnegative functions in $C^{\infty}(\bar{\Omega})$. For a finite collection of these functions $S_{j}=\left\{p_{k}\right\}_{k=1}^{j}$ the following optimal design problems are introduced

$$
\tilde{P}_{j}=\inf _{C(\underline{P}, x)} F(\phi),
$$

subject to the constraints:

$$
\int_{\Omega} p_{k}(x)|\nabla \phi|^{2} d x \leq K^{2} \int_{\Omega} p_{k}(x)(1+1 / j) d x, \text { for } p_{k} \text { in } S_{j}
$$


where $\phi$ is the $H_{0}^{1}(\Omega)$ solution to the state equation

$$
-\operatorname{div}(C(\underline{A}, x) \nabla \phi)=f .
$$

Its clear that as $j$ tends to infinity the constraints given in the design problems $\tilde{P}_{j}$ approach the constraint given by (37). The problems $\tilde{P}_{j}$ share the same feature as (35) in that they are optimal design problems over all configurations of $N$ dielectrics. The connection between the minimizer of the relaxed problem and minimizing sequences of configurations is given in the following theorem.

Theorem 1.6 Given a minimizer $\hat{C}^{\infty}(\underline{P}, x)$ of $Q R$ then there is a sequence of configurations and associated controls $\left\{C^{j}(\underline{P}, x)\right\}_{j=1}^{\infty}$ in $\mathcal{C}(\underline{A}, \underline{\gamma})$ such that for all $\underline{P}$ in $\mathcal{N}(\underline{A})$, the sequence $\left\{C^{j}(\underline{P}, x)\right\}_{j=1}^{\infty}$ G-converges to $\hat{C}^{\infty}(\underline{P}, x)$ and the state variables $\phi^{j}$ satisfy the constraints (46). For this case one has

$$
\lim _{j \rightarrow \infty} F\left(\phi^{j}\right)=Q R .
$$

Moreover, given any $\epsilon>0$ there exists an index $J>0$ such that for all $j>J$,

$$
\tilde{P}_{j} \leq F\left(\phi^{j}\right) \leq \tilde{P}_{j}+\epsilon
$$

and

$$
\lim _{j \rightarrow \infty} \tilde{P}_{j}=Q R
$$

Thus one can use the minimizer for the relaxed problem to recover nearly optimal constrained gradient designs of $N$ dielectrics.

The paper is organized as follows. In the next section the local formula for the derivative of the effective dielectric tensor viewed as a function of its constituent dielectric constants is derived together with a local homogenization theorem. In section three Theorem 1.2 is derived and the relaxation theorems are proved in section four. An overview of the results given here together with the recent results for problems where the design criteria is given in terms of the $L^{2}$ norm of the gradient are discussed in the conclusion.

\section{Local Formulas for the Derivative the G Limit and Local Homogenization}

In this section we prove Theorem (1.1). We consider a sequence $\left\{C^{n}(\underline{P}, x)\right\}_{n=1}^{\infty}$ that $\mathrm{G}$ converges to $C^{\infty}(\underline{P}, x)$ for every $\underline{P}$ in $\mathcal{N}(\underline{A})$. Given the sequence $\left\{C^{n}(\underline{A}, x)\right\}_{n=1}^{\infty}$, a vector $E$ in $R^{m}$ and an open set $\omega \subset \Omega$ we introduce the local corrector functions $\varphi_{E}^{n}$ where $\varphi_{E}^{n}$ is the $H_{0}^{1}(\omega)$ solution of

$$
\left.-\operatorname{div}\left(C^{n}(\underline{A}, x)\right)\left(\nabla \varphi_{E}^{n}+E\right)\right)=-\operatorname{div}\left(C^{\infty}(\underline{A}, x) E\right), \text { in } \omega .
$$

Equation (51) is written in the variational form given by

$$
\int_{\omega} C^{n}(\underline{A}, x)\left(\nabla \varphi_{E}^{n}+E\right) \cdot \nabla \psi d x-\int_{\omega}\left(C^{\infty}(\underline{A}, x) E\right) \cdot \nabla \psi d x=0,
$$


for all $\psi$ in $H_{0}^{1}(\omega)$. In the sequel a generic open subset of $\Omega$ is denoted by $\omega$. The measure of $\omega$ is denoted by $|\omega|$ and standard apriori estimates using Cauchy's inequality in (52) give

$$
\int_{\omega}\left|\nabla \varphi_{E}^{n}\right|^{2} d x \leq\left(4 \Lambda^{2} / \lambda^{2}\right)|\omega||E|^{2}
$$

and

$$
\int_{\omega}\left|\nabla \varphi_{E}^{n}+E\right|^{2} d x \leq\left(4 \Lambda^{2} / \lambda^{2}+1\right)|\omega||E|^{2} .
$$

We now list the convergence properties for the sequence of corrector functions.

Lemma 2.1 The sequence $\left\{\varphi_{E}^{n}\right\}_{n=1}^{\infty}$ converges weakly to zero in $H_{0}^{1}(\omega)$ thus

$$
\nabla \varphi_{E}^{n} \rightarrow 0, \text { weakly in } L^{2}(\omega)^{N},
$$

and

$$
C^{n}(\underline{A}, x)\left(\nabla \varphi_{E}^{n}+E\right) \rightarrow C^{\infty}(\underline{A}, x) E, \text { weakly in } L^{2}(\omega)^{N} .
$$

The proof is standard see (Ref. 10).

For $\delta \beta$ and $\underline{M}$ such that $\underline{A}+\delta \beta \underline{M}$ lies in $\mathcal{N}(\underline{A})$ consider the sequence $\left\{C^{n}(\underline{A}+\delta \beta \underline{M}, x)\right\}_{n=1}^{\infty}$. Here $\underline{M}$ is taken to be an array of dielectric tensors that are identically zero except for the $i^{t h}$ component dielectric tensor $M_{i}$. Here $\left|M_{i}\right|=\sqrt{\sum_{k, l}\left[M_{i}\right]_{k l}^{2}}$. The sequence of coefficients $\left\{C^{n}(\underline{A}+\delta \beta \underline{M}, x)\right\}_{n=1}^{\infty}$ differs from $\left\{C^{n}(\underline{A}, x)\right\}_{n=1}^{\infty}$ by the increment $\delta \beta M_{i} \chi_{i}^{n}$. The $\mathrm{G}$ limit for the sequence $\left\{C^{n}(\underline{A}+\delta \beta \underline{M}, x)\right\}_{n=1}^{\infty}$ is written as $C^{\infty}(\underline{A}+\delta \beta \underline{M}, x)$. We set $\delta C=C^{\infty}(\underline{A}+\delta \beta \underline{M}, x)-C^{\infty}(\underline{A}, x$,$) and examine the dependence of \delta C$ with respect to $\delta \beta$.

The first step is to examine the dependence of $(1 /|\omega|) \int_{\omega} \delta C E \cdot E d x$ with respect to the increment $\delta \beta$. The correctors associated with the sequence $\left\{C^{n}((\underline{A}+\delta \beta \underline{M}, x)\}_{n=1}^{\infty}\right.$ are denoted by $\hat{\varphi}_{E}^{n}+E \cdot x$ where $\hat{\varphi}_{E}^{n}$ are the $H_{0}^{1}(\omega)$ solutions of

$$
\int_{\omega} C^{n}\left((\underline{A}+\delta \beta \underline{M}, x)\left(\nabla \hat{\varphi}_{E}^{n}+E\right) \cdot \nabla \psi d x-\int_{\omega}\left(C^{\infty}(\underline{A}+\delta \beta \underline{M}, x) E\right) \cdot \nabla \psi d x=0,\right.
$$

for all $\psi$ in $H_{0}^{1}(\omega)$ and

$$
\begin{aligned}
C^{n}(\underline{A}+\delta \beta \underline{M}, x)\left(\nabla \hat{\varphi}_{E}^{n}+E\right) & \rightarrow C^{\infty}(\underline{A}+\delta \beta \underline{M}, x) E, \text { weakly in } L^{2}(\omega)^{N}, \\
\nabla \hat{\varphi}_{E}^{n} & \rightarrow 0, \text { weakly in } L^{2}(\omega)^{N} .
\end{aligned}
$$

For any choice of $p(x)$ in $C^{\infty}(\bar{\omega})$ it follows from (56) and (58) that

$$
\begin{aligned}
& (1 /|\omega|) \int_{\omega} p(x) \delta C E \cdot E d x \\
= & \lim _{n \rightarrow \infty}(1 /|\omega|) \int_{\omega} p(x)\left(C^{n}(\underline{A}+\delta \beta \underline{M}, x)\left(\nabla \hat{\varphi}_{E}^{n}+E\right)-C^{n}(\underline{A}, x)\left(\nabla \varphi_{E}^{n}+E\right)\right) \cdot E d x .
\end{aligned}
$$

Writing $C^{n}(\underline{A}+\delta \beta \underline{M}, x)=C^{n}(\underline{A}, x)+\delta \beta M_{i} \chi_{i}^{n}$ and $\hat{\varphi}_{E}^{n}=\varphi_{E}^{n}+\delta \varphi^{n}$, where $\delta \varphi^{n}=\hat{\varphi}_{E}^{n}-\varphi_{E}^{n}$ and substitution into (60) gives

$$
\begin{aligned}
& (1 /|\omega|) \int_{\omega} p \delta C E \cdot E d x \\
= & \lim _{n \rightarrow \infty}(1 /|\omega|) \int_{\omega} p \delta \beta M_{i} \chi_{i}^{n}\left(\nabla \varphi_{E}^{n}+E\right) \cdot E+p \delta \beta M_{i} \chi_{i}^{n} \nabla \delta \varphi^{n} \cdot E+p C^{n}(\underline{A}, x) \nabla \delta \varphi^{n} \cdot E d x .
\end{aligned}
$$


To proceed further we subtract (52) from (57) to obtain for every $\psi$ in $H_{0}^{1}(\omega)$ the equation

$$
\begin{aligned}
0 & =\int_{\omega} \delta \beta M_{i} \chi_{i}^{n}\left(\nabla \varphi_{E}^{n}+E\right) \cdot \nabla \psi d x+\int_{\omega} C^{n}(\underline{A}, x) \nabla \delta \varphi^{n} \cdot \nabla \psi d x \\
& +\int_{\omega} \delta \beta M_{i} \chi_{i}^{n} \nabla \delta \varphi^{n} \cdot \nabla \psi d x-\int_{\omega} \delta C E \cdot \nabla \psi d x .
\end{aligned}
$$

Choosing $\psi=\left(p \varphi_{E}^{n}\right)$ in (62) substitution into (61) and taking limits gives

$$
\begin{aligned}
& (1 /|\omega|) \int_{\omega} p \delta C E \cdot E d x \\
= & \delta \beta \lim _{n \rightarrow \infty}\left((1 /|\omega|) \int_{\omega} p M_{i} \chi_{i}^{n}\left(\nabla \varphi_{E}^{n}+E\right) \cdot\left(\nabla \varphi_{E}^{n}+E\right) d x\right. \\
+ & \left.(1 /|\omega|) \int_{\omega} p M_{i} \chi_{i}^{n}\left(\nabla \varphi_{E}^{n}+E\right) \cdot \nabla \delta \varphi^{n} d x\right) .
\end{aligned}
$$

Writing

$$
\begin{aligned}
\overline{\mathcal{S}}= & \limsup _{n \rightarrow \infty}(1 /|\omega|) \int_{\omega} p \delta \beta M_{i} \chi_{i}^{n}\left(\nabla \varphi_{E}^{n}+E\right) \cdot \nabla \delta \varphi^{n} d x \\
& \operatorname{and} \\
\underline{\mathcal{S}}= & \liminf _{n \rightarrow \infty}(1 /|\omega|) \int_{\omega} p \delta \beta M_{i} \chi_{i}^{n}\left(\nabla \varphi_{E}^{n}+E\right) \cdot \nabla \delta \varphi^{n} d x,
\end{aligned}
$$

standard apriori estimates are used to obtain

$$
|\overline{\mathcal{S}}| \leq\|p\|_{\infty} K|E|^{2} \sqrt{|\delta \beta|} \text { and }|\underline{\mathcal{S}}| \leq\|p\|_{\infty} K|E|^{2} \sqrt{|\delta \beta|},
$$

where $K$ is a constant independent of $\delta \beta$ and choice of $\omega \subset \Omega$ and $\|p\|_{\infty}$ is the maximum value of $p$ on $\bar{\omega}$. We establish the following

Lemma 2.2 For every $\omega \subset \Omega$ and function $p$ in $C^{\infty}(\bar{\omega})$, the limit

$$
\lim _{n \rightarrow \infty}\left((1 /|\omega|) \int_{\omega} p M_{i} \chi_{i}^{n}\left(\nabla \varphi_{E}^{n}+E\right) \cdot\left(\nabla \varphi_{E}^{n}+E\right) d x\right)
$$

exists and

$$
\begin{aligned}
& (1 /|\omega|) \int_{\omega} p \delta C E \cdot E d x \\
= & \delta \beta \lim _{n \rightarrow \infty}\left((1 /|\omega|) \int_{\omega} p M_{i} \chi_{i}^{n}\left(\nabla \varphi_{E}^{n}+E\right) \cdot\left(\nabla \varphi_{E}^{n}+E\right) d x\right)+\delta \beta R(\delta \beta, \omega, p),
\end{aligned}
$$

where

$$
|R(\delta \beta, \omega, p)| \leq\|p\|_{\infty} K|E|^{2} \sqrt{|\delta \beta|}
$$

Proof. We put

$$
\begin{aligned}
H_{n}= & \left((1 /|\omega|) \int_{\omega} p M_{i} \chi_{i}^{n}\left(\nabla \varphi_{E}^{n}+E\right) \cdot\left(\nabla \varphi_{E}^{n}+E\right) d x\right) \\
& \text { and } \\
R_{n}= & \left((1 /|\omega|) \int_{\omega} p M_{i} \chi_{i}^{n}\left(\nabla \varphi_{E}^{n}+E\right) \cdot \nabla \delta \varphi^{n} d x\right)
\end{aligned}
$$


and from (54) we see that $\bar{H}=\limsup _{n \rightarrow \infty} H_{n}$ and $\underline{H}=\liminf _{n \rightarrow \infty} H_{n}$ are finite. Passing to subsequences as necessary we deduce that

$$
(1 /|\omega|) \int_{\omega} p \delta C E \cdot E d x=\delta \beta \bar{H}+\delta \beta \bar{R}(\delta \beta, \omega, p),
$$

where for each $\delta \beta, \bar{R}(\delta \beta, \omega, p)$ is a cluster point of the sequence $\left\{R_{n}\right\}_{n=1}^{\infty}$ and from (65) it is evident that $\bar{R}(\delta \beta, \omega, p) \leq\|p\|_{\infty} K|E|^{2} \sqrt{|\delta \beta|}$. Similar considerations give

$$
(1 /|\omega|) \int_{\omega} p \delta C E \cdot E d x=\delta \beta \underline{H}+\delta \beta \underline{R}(\delta \beta, \omega, p),
$$

where $\underline{R}(\delta \beta, \omega, p)$ is a cluster point of the sequence $\left\{R_{n}\right\}_{n=1}^{\infty}$ and $\underline{R}(\delta \beta, \omega, p) \leq\|p\|_{\infty} K|E|^{2} \sqrt{|\delta \beta|}$. It is evident from (70) and (71) that $\underline{H}=\bar{H}$. From this we deduce $\underline{R}(\delta \beta, \omega, p)=\bar{R}(\delta \beta, \omega, p) \triangleq$ $R(\delta \beta, \omega, p)$ and the Lemma is established.

With Lemma 2.2 in hand we establish Theorem 1.1. We consider subsets $\omega$ of $\Omega$ given by cubes $Q(x, r)$ and the associated local corrector functions are denoted by $\varphi_{E}^{n, r}$. We put

$$
H(x, r)=\lim _{n \rightarrow \infty}(1 /|Q(x, r)|) \int_{Q(x, r)} M_{i} \chi_{i}^{n}\left(\nabla \varphi_{E}^{n, r}+E\right) \cdot\left(\nabla \varphi_{E}^{n, r}+E\right) d y .
$$

and we introduce the functions $\bar{f}(x)$ and $\underline{f}(x)$ defined everywhere in $\Omega$ by

$$
\bar{f}(x) \triangleq \limsup _{r \rightarrow 0} H(x, r)
$$

and

$$
\underline{f}(x) \triangleq \liminf _{r \rightarrow 0} H(x, r)
$$

The estimate (54) shows that these functions are bounded on $\Omega$. Given the increment $\delta \beta$ we consider the intersection of Lebesgue points of $C^{\infty}(\underline{A}, x)$ and $C^{\infty}(\underline{A}+\delta \beta \underline{M}, x)$. We choose $p=1$ and from Lemma 2.2 we have the following identity

$$
\begin{aligned}
\delta C E \cdot E & =\lim _{r \rightarrow 0}(1 /|Q(x, r)|) \int_{Q(x, r)} \delta C E \cdot E d y \\
& =\delta \beta \lim _{r \rightarrow 0}(H(x, r)+R(\delta \beta, Q(x, r), 1))
\end{aligned}
$$

almost everywhere on $\Omega$ and for any $E$ in $R^{m}$. Passing to subsequences as necessary one has

$$
\delta C E \cdot E=\delta \beta(\bar{f}(x)+\bar{R}(\delta \beta, x)) \text {, a.e. on } \Omega,
$$

where $\bar{R}(\delta \beta, x)$ is a cluster point of the sequence $\{R(\delta \beta, Q(x, r), 1)\}_{r>0}$ and $|\bar{R}(\delta \beta, x)| \leq$ $K|E|^{2} \mid \sqrt{|\delta \beta|}$. A similar consideration shows that

$$
\delta C E \cdot E=\delta \beta(\underline{f}(x)+\underline{R}(\delta \beta, x)), \text { a.e. on } \Omega
$$


where $\underline{R}(\delta \beta, x)$ is a cluster point of the sequence $\{R(\delta \beta, B(x, r), 1)\}_{r>0}$ and $|\underline{R}(\delta \beta, x)| \leq$ $K|E|^{2} \sqrt{|\delta \beta|}$. We subtract (77) from (76) to obtain

$$
\bar{f}(x)-\underline{f}(x)=\underline{R}(\delta \beta, x)-\bar{R}(\delta \beta, x), \text { a.e.on } \Omega,
$$

to see that

$$
|\bar{f}(x)-\underline{f}(x)|<2 K|E|^{2} \sqrt{|\delta \beta|} \text {, a.e. on } \Omega .
$$

Since $\bar{f}(x)$ and $\underline{f}(x)$ are independent of the increment $\delta \beta$ we deduce that

$$
\lim _{r \rightarrow 0} \lim _{n \rightarrow \infty}(1 /|Q(x, r)|) \int_{Q(x, r)} M_{i} \chi_{i}^{n}\left(\nabla \varphi_{E}^{n, r}+E\right) \cdot\left(\nabla \varphi_{E}^{n, r}+E\right) d y
$$

exists almost everywhere on $\Omega$. In this way it is seen that $\underline{R}(\delta \beta, x)=\bar{R}(\delta \beta, x) \triangleq R(\delta \beta, x)$ and

$\delta C E \cdot E=\delta \beta \lim _{r \rightarrow 0} \lim _{n \rightarrow \infty}(1 /|Q(x, r)|) \int_{Q(x, r)} M_{i} \chi_{i}^{n}\left(\nabla \varphi_{E}^{n, r}+E\right) \cdot\left(\nabla \varphi_{E}^{n, r}+E\right) d y+\delta \beta R(\delta \beta, x)$,

a.e. on $\Omega$, where $R(\delta \beta, x)<K|E|^{2} \sqrt{|\delta \beta|}$. It is evident from (81) that $\frac{\partial C^{\infty}}{\partial M_{i}} E \cdot E$ exists and is given by

$$
\frac{\partial C^{\infty}}{\partial M_{i}} E \cdot E=\lim _{r \rightarrow 0} \lim _{n \rightarrow \infty}(1 /|Q(x, r)|) \int_{Q(x, r)} M_{i} \chi_{i}^{n}\left(\nabla \varphi_{E}^{n, r}+E\right) \cdot\left(\nabla \varphi_{E}^{n, r}+E\right) d y
$$

and Theorem 1.1 follows.

Collecting results, we substitute (81) into (67) of Lemma 2.3 to obtain the local homogenization theorem.

Theorem 2.1 Local Homogenization Theorem. Under the hypotheses of Theorem 1.1, given any $\omega \subset \Omega$ and $p$ in $C^{\infty}(\bar{\omega})$ the sequence of local correctors, $\left\{\varphi_{E}^{n}+E \cdot x\right\}_{n=1}^{\infty}$ defined by (51) satisfy

$$
\lim _{n \rightarrow \infty}(1 /|\omega|) \int_{\omega} p M_{i} \chi_{i}^{n}\left(\nabla \varphi_{E}^{n}+E\right) \cdot\left(\nabla \varphi_{E}^{n}+E\right) d x=(1 /|\omega|) \int_{\omega} p \frac{\partial C^{\infty}}{\partial M_{i}} E \cdot E d x .
$$

For future reference it is noted that the estimate (54) gives the bound

$$
\sigma(x) E \cdot E \leq\left(4 \Lambda^{2} / \lambda^{2}\right)|E|^{2},
$$

almost everywhere in $\Omega$ for $E$ in $R^{m}$.

\section{Homogenization of Products of Weakly Converging Sequences of Gradients}

In this Section Theorem 1.1 and Theorem 2.1 are applied together with a localization argument to establish Theorem 1.2. Recall that the sequences of solutions of (15) denoted by 
$\left\{\phi^{n}\right\}_{n=1}^{\infty}$ converge weakly to the solution $\phi^{\infty}$ of (16) and the associated sequence of coefficient matrices $\left\{C^{n}(\underline{P}, x)\right\}_{n=1}^{\infty} \mathrm{G}$ converge to $C^{\infty}(\underline{P}, x)$ for every $\underline{P}$ in $\mathcal{N}(\underline{A})$.

The first step in the proof is to approximate $\phi^{\infty}$ by piecewise affine functions. Given $\epsilon>0$ there exists a function $w^{\epsilon}$ in $H_{0}^{1}(\Omega)$ which is piecewise affine on $\Omega$ and

$$
\int_{\Omega}\left|\nabla w^{\epsilon}-\nabla \phi^{\infty}\right|^{2} d x<\epsilon^{2}
$$

see for example (Ref. 18). The gradient of $w^{\epsilon}$ is constant on the open sets $\omega_{\epsilon}^{i}$ and $\bar{\Omega}=\cup_{i=1}^{\kappa(\epsilon)} \bar{\omega}_{\epsilon}^{i}$. In each open set $\omega_{\epsilon}^{i}$, one has $w^{\epsilon}=E^{i} \cdot x+c^{i}$, where $E^{i}$ is a constant vector in $R^{m}$ and $c^{i}$ is a constant.

The global corrector $\left\{\phi^{n, \epsilon}\right\}_{n=1}^{\infty}$ is introduced. The right-hand side $f^{\epsilon}=-\operatorname{div}\left(C^{\infty}(\underline{A}, x) \nabla w^{\epsilon}\right)$, is chosen and $\phi^{n, \epsilon}$ is defined to be the $H_{0}^{1}(\Omega)$ solution of

$$
-\operatorname{div}\left(C^{n}(\underline{A}, x) \nabla \phi^{n, \epsilon}\right)=f^{\epsilon} .
$$

One easily obtains the following convergence properties given by

Lemma 3.1 For $\epsilon$ fixed,

$$
\phi^{n, \epsilon} \rightarrow w^{\epsilon}, \text { as } n \rightarrow \infty \text { in } H_{0}^{1}(\Omega) \text { and } C^{n}(\underline{A}, x) \nabla \phi^{n, \epsilon} \rightarrow C^{\infty}(\underline{A}, x) \nabla w^{\epsilon} \text { in } L^{2}(\Omega)^{m} .
$$

The error between the weakly converging sequences $\left\{\phi^{n}\right\}_{n=1}^{\infty}$ and the sequence of global correctors $\left\{\phi^{n, \epsilon}\right\}_{n=1}^{\infty}$ can be controlled uniformly with respect to $n$. This is made precise in the following Lemma.

Lemma 3.2 There exists a constant $C_{0}$ independent of $n$ and $\epsilon$ such that

$$
\int_{\Omega}\left|\nabla \phi^{n}-\nabla \phi^{n, \epsilon}\right|^{2} d x \leq C_{0}^{2}(\Lambda / \lambda)^{2} \epsilon^{2}, \text { for all } n>0
$$

Proof. We start by showing $\left\|f^{\epsilon}-f\right\|_{H^{-1}(\Omega)} \leq \epsilon \Lambda$. Given $\psi$ in $H_{0}^{1}(\Omega)$ we have

$$
\begin{aligned}
\left|<_{H^{-1}}\left(f^{\epsilon}-f\right), \psi>_{H_{0}^{1}}\right| & =\left|\int_{\Omega} C^{\infty}(\underline{A}, x)\left(\nabla w^{\epsilon}-\nabla \phi\right) \cdot \nabla \psi d x\right| \\
& \leq \Lambda \int_{\Omega}\left|\nabla w^{\epsilon}-\nabla \phi\right||\nabla \psi|, d x \\
& \leq \epsilon \Lambda\|\psi\|_{H_{0}^{1}(\Omega)},
\end{aligned}
$$

and the claim follows. Next one has the estimate given by

$$
\begin{aligned}
\lambda \int_{\Omega}\left|\nabla \phi^{n}-\nabla \phi^{n, \epsilon}\right|^{2} d x & \leq \int_{\Omega} C^{n}(\underline{A}, x)\left(\nabla \phi^{n}-\nabla \phi^{n, \epsilon}\right) \cdot\left(\nabla \phi^{n}-\nabla \phi^{n, \epsilon}\right) d x \\
& \leq\left\|f^{\epsilon}-f\right\|_{H^{-1}} C_{0} \sqrt{\int_{\Omega}\left|\nabla \phi^{n}-\nabla \phi^{n, \epsilon}\right|^{2} d x}
\end{aligned}
$$

where $C_{0}$ depends only on $\Omega$ and $m$ and comes from the Poincare Friedrichs inequality. The inequality (88) follows noting that $\left\|f^{\epsilon}-f\right\|_{H^{-1}} \leq \Lambda \epsilon$. 
Collecting results we write $\phi=w^{\epsilon}+r^{\epsilon}, \phi^{n}=\phi^{n, \epsilon}+z^{n, \epsilon}$. Here $r^{\epsilon}$ and $z^{n, \epsilon}$ are in $H_{0}^{1}(\Omega)$ and satisfy

$$
\int_{\Omega}\left|\nabla r^{\epsilon}\right|^{2} d x<\epsilon^{2} \text { and } \int_{\Omega}\left|\nabla z^{n, \epsilon}\right|^{2} d x<\epsilon^{2}, \text { for all } n>0 .
$$

Given $p(x)$ in $C^{\infty}(\bar{\Omega})$, one observes that $1=\sum_{j=1}^{N} \chi_{j}^{n}(x)$ and writes

$$
\begin{aligned}
& \int_{\Omega} p\left|\nabla \phi^{n}\right|^{2} d x \\
= & \int_{\Omega} p\left(\sum_{j=1}^{N} \chi_{j}^{n}(x)\right) \mid \nabla\left(\phi^{n, \epsilon}+\left.z^{n, \epsilon}\right|^{2}\right) d x \\
= & \sum_{i=1}^{\kappa(\epsilon)} \sum_{j=1}^{N} \int_{\omega_{\epsilon}^{i}} p \chi_{j}^{n}\left|\nabla \phi^{n, \epsilon}\right|^{2} d x+O(\epsilon) .
\end{aligned}
$$

The lemma that gives the connection between global and local correctors is provided by the following localization lemma.

Lemma 3.3 Localization Lemma. Let $\varphi_{E^{i}}^{i, n, \epsilon}$ be the $H_{0}^{1}\left(\omega_{\epsilon}^{i}\right)$ solution of

$$
\left.-\operatorname{div}\left(C^{n}(\underline{A}, x)\right)\left(\nabla \varphi_{E^{i}}^{i, n}+E^{i}\right)\right)=-\operatorname{div}\left(C^{\infty}(\underline{A}, x) E^{i}\right),
$$

then

$$
\lim _{n \rightarrow \infty} \int_{\omega_{\epsilon}^{i}}\left|\nabla \varphi_{E^{i}}^{i, n, \epsilon}+E^{i}-\nabla \phi^{n, \epsilon}\right|^{2} d x=0
$$

Proof. From the properties of the local corrector introduced in Section 2 one has $\varphi_{E^{i}}^{i, n, \epsilon} \rightarrow 0$, weakly in $H_{0}^{1}\left(\omega_{\epsilon}^{i}\right)$ and $C^{n}(\underline{A}, x)\left(\nabla \varphi_{E^{i}}^{i, n, \epsilon}+E^{i}\right) \rightarrow C^{\infty}(\underline{A}, x) E^{i}$, weakly in $L^{2}\left(\omega_{\epsilon}^{i}\right)^{m}$.

The properties of the global corrector $\phi^{n, \epsilon}$ imply that it is a solution of

$$
-\operatorname{div}\left(C^{n}(\underline{A}, x) \nabla \phi^{n, \epsilon}\right)=-\operatorname{div}\left(C^{\infty}(\underline{A}, x) E^{i}\right), \text { on } \omega_{\epsilon}^{i},
$$

i.e., for all $\psi$ in $H_{0}^{1}\left(\omega_{\epsilon}^{i}\right)$

$$
\int_{\omega_{\epsilon}^{i}} C^{n}(\underline{A}, x) \nabla \phi^{n, \epsilon} \cdot \nabla \psi d x=\int_{\omega_{\epsilon}^{i}} C^{\infty}(\underline{A}, x) E^{i} \cdot \nabla \psi d x,
$$

and

$$
\begin{aligned}
& \phi^{n, \epsilon} \rightarrow w^{\epsilon}=E^{i} \cdot x+c^{i}, \text { weakly in } H^{1}\left(\omega_{\epsilon}^{i}\right), \\
& C^{n}(\underline{A}, x) \nabla \phi^{n, \epsilon} \rightarrow C^{\infty}(\underline{A}, x) E^{i}, \text { weakly in } L^{2}\left(\omega_{\epsilon}^{i}\right)^{m} .
\end{aligned}
$$

The Lemma now follows immediately from Remark 1 of Section 10 in (Ref. 10). 
The local homogenization Theorem 2.1 and the localization Lemma 3.3 are applied and we work up from small to intermediate scales to find that

$$
\begin{aligned}
\sum_{i=1}^{\kappa(\epsilon)} \int_{\omega_{\epsilon}^{i}} p(\sigma(x)+I) E^{i} \cdot E^{i} d x & =\sum_{i=1}^{\kappa(\epsilon)} \sum_{j=1}^{N} \int_{\omega_{\epsilon}^{i}} p \nabla_{k k}^{j} C^{\infty}(\underline{A},(x)) E^{i} \cdot E^{i} d x \\
& =\sum_{i=1}^{\kappa(\epsilon)} \sum_{i=j}^{N} \lim _{n \rightarrow \infty} \int_{\omega_{\epsilon}^{i}} p \chi_{j}^{n}\left|\nabla \varphi_{E^{i}}^{i, n, \epsilon}+E^{i}\right|^{2} d x \\
& =\lim _{n \rightarrow \infty} \sum_{i=1}^{\kappa(\epsilon)} \sum_{j=1}^{N} \int_{\omega_{\epsilon}^{i}} p \chi_{j}^{n}\left|\nabla \phi^{n, \epsilon}\right|^{2} d x
\end{aligned}
$$

Proceeding from large scales down to intermediate scales we see that

$$
\begin{aligned}
\lim _{n \rightarrow \infty} \int_{\Omega} p\left|\nabla \phi^{n}\right|^{2} d x & =\lim _{n \rightarrow \infty} \sum_{i=1}^{\kappa(\epsilon)} \int_{\omega_{\epsilon}^{i}} p\left|\nabla \phi^{n, \epsilon}\right|^{2} d x+O(\epsilon) \\
& =\sum_{i=1}^{\kappa(\epsilon)} \int_{\omega_{\epsilon}^{i}} p(\sigma(x)+I) E^{i} \cdot E^{i} d x+O(\epsilon) \\
& =\int_{\Omega} p(\sigma(x)+I) \nabla w^{\epsilon} \cdot \nabla w^{\epsilon} d x+O(\epsilon) .
\end{aligned}
$$

Since $\epsilon$ is arbitrary and recalling (84) we obtain the desired result given by

$$
\lim _{n \rightarrow \infty} \int_{\Omega} p\left|\nabla \phi^{n}\right|^{2} d x=\int_{\Omega} p(\sigma(x)+I) \nabla \phi^{\infty} \cdot \nabla \phi^{\infty} d x
$$

and Theorem 1.2 follows.

\section{Relaxation}

In this section the properties of the relaxed problem (38) given by Theorems 1.5 and 1.6 are established. The proofs of Theorems 1.3 and 1.4 follow identical lines. In establishing the properties of relaxed problem the following compactness result will be used.

Theorem 4.1 Compactness Property. Given any sequence $\left\{C^{n}(\underline{P}, x)\right\}_{n=1}^{\infty}$ in $\mathcal{C}(\underline{A}, \underline{\gamma})$, there exists a subsequence $\left\{C^{n^{\prime}}(\underline{P}, x)\right\}_{n^{\prime}=1}^{\infty}$ and a function $C^{\infty}(\underline{P}, x)$ in $\mathcal{G C}(\underline{A}, \underline{\gamma})$ such that $\bar{C}^{n^{\prime}}(\underline{P}, x)$ G converges to $C^{\infty}(\underline{P}, x)$ for all $\underline{P}$ in $\mathcal{N}(\underline{A})$.

This property follows immediately from (Ref. 13).

A special sequence of controls $\left\{C^{n}(\underline{P}, x)\right\}_{n=1}^{\infty}$ in $\mathcal{C}(\underline{A}, \underline{\gamma})$ is constructed. This sequence will be used to establish Theorems 1.5 and 1.6. To construct this sequence consider a minimizing sequence $\left\{C^{\infty, n}(\underline{P}, x)\right\}_{n=1}^{\infty}$ for $Q R$. The associated set of electric potentials for the sequence $\left\{C^{\infty, n}(\underline{A}, x)\right\}_{n=1}^{\infty}$ is denoted by $\left\{\phi^{n}\right\}_{n=1}^{\infty}$ and $Q R=\lim _{n \rightarrow \infty} F\left(\phi^{n}\right)$.

For each $n$ there exists a sequence of controls $\left\{C^{n, k}(\underline{P}, x)\right\}_{k=1}^{\infty}$ in $\mathcal{C}(\underline{A}, \gamma)$ such that $C^{n, k}(\underline{P}, x), \mathrm{G}$ converges to $C^{\infty, n}(\underline{P}, x)$ for every $\underline{P}$ in $\mathcal{N}(\underline{A})$. The associated potentials $\phi^{n, k}$ in $H_{0}^{1}(\Omega)$ satisfy the state equation

$$
-\operatorname{div}\left(C^{n, k}(\underline{A}, x) \nabla \phi^{n, k}\right)=f
$$


and for every nonnegative $p$ in $C^{\infty}(\bar{\Omega})$ the homogenization theorem 1.2 gives

$$
\lim _{k \rightarrow \infty} \int_{\Omega} p(x)\left|\nabla \phi^{n, k}\right|^{2} d x=\int_{\Omega} p(x)\left(\sigma^{n}(x)+I\right) \nabla \phi^{n} \cdot \nabla \phi^{n} d x \leq K^{2} \int_{\Omega} p(x) d x,
$$

where the covariance matrix $\sigma^{n}(x)$ is

$$
\sigma^{n}(x) E \cdot E=\sum_{i=1}^{N}\left(\nabla_{k k}^{i} C^{\infty, n}(\underline{A}, x)\right) E \cdot E-|E|^{2},
$$

for all vectors $E$ in $R^{m}$. A countable dense subset $\left\{p_{\ell}\right\}_{\ell=1}^{\infty}$ of the set of nonnegative functions in $C^{\infty}(\bar{\Omega})$ is introduced. Given $n$ put $S_{n}=\left\{p_{\ell}\right\}_{\ell=1}^{n}$ then there exists an index $k_{n}$ for which

$$
\begin{gathered}
\int_{\Omega} p_{\ell}\left|\nabla \phi^{n, k_{n}}\right|^{2} d x \leq K^{2} \int_{\Omega} p_{\ell}(1+1 / n) d x, \text { for every } p_{\ell} \text { in } S_{n} \\
\left|F\left(\phi^{n, k_{n}}\right)-F\left(\phi^{n}\right)\right|<1 / n
\end{gathered}
$$

and

$$
-\operatorname{div}\left(C^{n, k_{n}}(\underline{A}, x) \nabla \phi^{n, k_{n}}\right)=f .
$$

Passing to a subsequence if necessary and appealing to the compactness property there exists a function $\tilde{C}^{\infty}(\underline{P}, x)$ in $\mathcal{G C}(\underline{A}, \underline{\gamma})$ for which

$$
\left\{C^{n, k_{n}}(\underline{P}, x)\right\}_{n=1}^{\infty}, \mathrm{G} \text { converges to } \tilde{C}^{\infty}(\underline{P}, x)
$$

for all $\underline{P}$ in $\mathcal{N}(\underline{A})$.

Definition 4.1 The sequence $\left\{\left(C^{n, k_{n}}(\underline{P}, x)\right\}_{n=1}^{\infty}\right.$ constructed above satisfying $(105-108)$ is called a configuration minimizing sequence.

The potential associated with the control $\tilde{C}^{\infty}(\underline{P}, x)$ is denoted by $\tilde{\phi}$ and

$$
-\operatorname{div}\left(\tilde{C}^{\infty}(\underline{A}, x) \nabla \tilde{\phi}\right)=f .
$$

We proceed to establish Theorem 1.5. For each $p_{\ell}$ in $\left\{p_{\ell}\right\}_{\ell=1}^{\infty}$ it follows that

$$
\begin{aligned}
\int_{\Omega} p_{\ell}(x)(\tilde{\sigma}(x)+I) \nabla \tilde{\phi} \cdot \nabla \tilde{\phi} d x & =\lim _{n \rightarrow \infty} \int_{\Omega} p_{\ell}\left|\nabla \phi^{n, k_{n}}\right|^{2} d x \\
& \leq K^{2} \int_{\Omega} p_{\ell} d x
\end{aligned}
$$

where

$$
\tilde{\sigma}(x) E \cdot E=\sum_{i=1}^{N}\left(\nabla_{k k}^{i} \tilde{C}^{\infty}(\underline{A}, x)\right) E \cdot E-|E|^{2}
$$

for every $E$ in $R^{m}$. Thus by density, for any nonnegative $p$ in $C^{\infty}(\bar{\Omega})$ one has

$$
\int_{\Omega} p(x)(\tilde{\sigma}(x)+I) \nabla \tilde{\phi} \cdot \nabla \tilde{\phi} d x \leq K^{2} \int_{\Omega} p(x) d x
$$


and $\tilde{C}^{\infty}(\underline{P}, x)$ is an admissible control for $Q R$. It is evident from the continuity of the objective function and (106) that $F(\tilde{\phi})=Q R$ and Theorem 1.5 follows.

To prove Theorem 1.6 one considers the design problems $\tilde{P}_{j}$ given by (45) and starts by showing $\tilde{P}_{j} \leq Q R$. For the configuration minimizing sequence $\left\{\left(C^{n, k_{n}}(\underline{P}, x)\right\}_{n=1}^{\infty}\right.$ of Definition 4.1 it is evident that given the index $j$ one has that for all $n>j$, that $C^{n, k_{n}}(\underline{P}, x)$ is admissable for $\tilde{P}_{j}$, and $\tilde{P}_{j} \leq F\left(\phi^{n, k_{n}}\right)$. Sending $n$ to infinity shows that $\tilde{P}_{j} \leq Q R$. Noting that $\tilde{P}_{j}$ is monotone increasing with $j$ and bounded above implies the existence of $\lim _{j \rightarrow \infty} \tilde{P}_{j}$. Next it is shown that $Q R=\lim _{j \rightarrow \infty} \tilde{P}_{j}$. Given $j>0$ one can choose a design denoted by $C^{j}(\underline{P}, x)$ with associated electric potential $\phi^{j}$ in $H_{0}^{1}(\Omega)$ for which

$$
\int_{\Omega} p_{k}(x)\left|\nabla \phi^{j}\right|^{2} d x \leq K^{2} \int_{\Omega} p_{k}(x)(1+1 / j) d x, \text { for } p_{k} \text { in } S_{j}
$$

and

$$
-\operatorname{div}\left(C^{j}(\underline{A}, x) \nabla \phi^{j}\right)=f .
$$

Where

$$
\tilde{P}_{j} \leq F\left(\phi^{j}\right) \leq \tilde{P}_{j}+1 / j
$$

From the compactness property and passage to subsequences if necessary there exists a function $\bar{C}^{\infty}(\underline{P}, x)$ in $\mathcal{G C}(\underline{A}, \underline{\gamma})$ such that $C^{j}(\underline{P}, x)$ G converges to $\bar{C}^{\infty}(\underline{P}, x)$ for for all $\underline{P}$ in $\mathcal{N}(\underline{A})$. The associated potential $\bar{\phi}$ in $H_{0}^{1}(\Omega)$ solves

$$
-\operatorname{div}\left(\bar{C}^{\infty}(\underline{A}, x) \nabla \bar{\phi}\right)=f .
$$

Here $\lim _{j \rightarrow \infty} F\left(\phi^{j}\right)=F(\bar{\phi})$ and arguing as above the associated covariance $\bar{\sigma}$ satisfies

$$
\int_{\Omega} p(x)(\bar{\sigma}(x)+I) \nabla \bar{\phi} \cdot \nabla \bar{\phi} d x \leq K^{2} \int_{\Omega} p(x) d x,
$$

for every $p$ in $C^{\infty}(\bar{\Omega})$. Thus $\bar{C}^{\infty}(\underline{P}, x)$ is an admissible design for $Q R$. Observing that $Q R \leq F(\bar{\phi})=\lim _{j \rightarrow \infty} F\left(\phi^{j}\right)$ and (115) gives the set of inequalities

$$
Q R \leq \lim _{j \rightarrow \infty} F\left(\phi^{j}\right) \leq \lim _{j \rightarrow \infty} \tilde{P}_{j} \leq Q R
$$

and one concludes that $Q R=\lim _{j \rightarrow \infty} \tilde{P}_{j}$. Lastly (49) is established. Consider the configuration minimizing sequence $\left\{\left(C^{n, k_{n}}(\underline{P}, x)\right\}_{n=1}^{\infty}\right.$. It is evident that the control $C^{j, k_{j}}(\underline{P}, x)$ is admissible for $\tilde{P}_{j}$ thus

$$
P_{j} \leq F\left(\phi^{j, k_{j}}\right) .
$$

The upper inequality in (49) follows recalling that $\lim _{j \rightarrow \infty} F\left(\phi^{j, k_{j}}\right)=Q R$ where $\phi^{j, k_{j}}$ is the state variable associated with $C^{j, k_{j}}(\underline{A}, x)$ and on noting that $\lim _{j \rightarrow \infty} \tilde{P}_{j}=Q R$.

\section{Conclusions}

The results given here are intended to provide the underpinnings for numerical methods for the design of multi-phase composites for optimal performance subject to constraints on 
the gradient of the state variable. Future work will apply the theory developed here to the numerical design of functionally graded materials.

It should be pointed out that for the case of two phase mixtures with one component having a nonzero dielectric constant and the other being zero, the comprehensive theory developed in (Ref. 19) provides a relaxed formulation for the design problem when the constraint is given in terms of the $L^{2}$ norm of the gradient. Here the fact that one of the dielectrics is zero delivers an optimal design problem of self adjoint type. However when both dielectric constants are nonzero the problem becomes non self adjoint and a design problem of the kind treated here is recovered.

Recently problems involving design criteria given directly in terms of the $L^{2}$ norm of the gradient have been addressed (Refs. 20-25). Here the objective is to force the gradient to be as close as possible to a target field in the $L^{2}$ norm. This problem is one of minimizing the "mean-square deviation of the gradient of the state." Gradient based methods for the numerical identification of minimizing sequences of configurations are given in (Ref. 20), while the full relaxation for the problem has recently been found in (Ref. 21). In (Ref. 22) a philosophy similar to (Ref. 19) is adopted and the new concept of constrained quaziconvex envelope is introduced. The constrained quaziconvex envelope is computed explicitly in (Ref. 23) for this problem. The approaches taken in (Ref. 21) and Ref. 23) are very different however both show that the only minimizing sequences for the the mean-square deviation of the gradient of the state are the well known rank one layered microstructures. The earlier work given in (Ref. 24) indicates the existence of a dense class of targets for which relaxation is accomplished through computation of the strong $L^{2}$ closure of the set of controls. The explicit representation of this set is given in (Ref. 24). That analysis also shows that minimizing sequences are given by rank one microstructures. For the case of $N$ anisotropic conductors the strong $L^{2}$ closure is characterized in (Ref. 25).

\section{References}

1. Nelson, J. K., Some Steps Toward the Automation of the Design of Composite Dielectric Structures, IEEE Transactions on Dielectrics and Electrical Insulaton, Vol. 1, pp. 663-671, 1994.

2. Li, Y. S., and Duxbury, P. M.. From Moduli Scaling to Breakdown Scaling: A Moment Spectrum Analysis, Physical Review, Vol. 40B, pp. 4889-4897, 1989.

3. Cabib, E., and Dal Maso, G., On a Class of Optimum Problems in Structural Design, Journal of Optimization Theory and Applications, Vol. 56, pp. 39-65, 1988.

4. Lurie, K. A., On the Optimal Distribution of the Resistivity Tensor of the Working Substance in a Magnetohydrodynamic Channel, Journal of Applied Mathematics and Mechanics. Vol. 34, pp. 255-274, 1970.

5. Murat, F., Un Contre-Example pour le Problème du Contrôle dans les Coefficients, Comptes Rendus de l' Academie des Sciences, Paris, Vol. 273A, pp. 708-711, 1971.

6. Rozvany, I. N., Olhoff, N., Cheng, K. T., and Taylor, J. E. On the Solid Plate Paradox in Structural Optimization, Journal of Structural Mechanics, Vol. 10, pp. 1-32, 1982. 
7. Lurie, K. A., and Cherkaev, A. V., Effective Characteristics of Composite Materials and the Optimal Design of Structural Elements, Uspekhi Mekhaniki (Advances in Mechanics), Vol. 9, pp. 3-81, 1986.

8. Murat, F., and Tartar, L., Calcul des Variations et Homogénéisation, Les Méthodes de l'Homogénéisation: Théorie et Applications en Physique, Edited by D. Bergman et. al., Collection de la Direction des Études et Recherches d'Electricité de France, Eyrolles, Paris, France, Vol. 57 pp. 319-369, 1985.

9. Spagnolo, S., Convergence in Energy for Elliptic Operators, Proceedings of the 3rd Symposium on Numerical Solutions of Partial Differential Equations, College Park, Maryland, 1975; Edited by B. Hubbard, Academic Press, New York, NY, pp. 469-498, 1976.

10. Murat, F., and Tartar, L., H-Convergence, Topics in the Mathematical Modelling of Composite Materials, Edited by A. Cherkaev and R. Kohn, Berkhauser, Boston, Massachusetts, pp. 21-43, 1997.

11. Dal Maso, G., An Introduction to $\Gamma$-Convergence, Birkhäuser, Boston, Massachusetts, 1993.

12. Bendsoe, M. P., Optimization of Structural Topology, Shape, and Material, Springer, Berlin, Germany, 1995.

13. Tartar, L., An Introduction to the Homogenization Method In Optimal Design, Springer Lecture Notes in Mathematics, Springer, Berlin, Germany, Vol. 1740, pp. 45-156, 2000.

14. Bergman, D. J., The Dielectric Constant of a Composite Material: A Problem in Classical Physics, Physics Reports, Vol. 43C, pp. 377-407, 1978.

15. Lipton, R., Optimal Bounds on Electric Field Fluctuations for Random Composites, Journal of Applied Physics, Vol. 88, pp. 4287-4296, 2000.

16. Lipton, R., Optimal Bounds on Field Fluctuations for Random Composites: ThreeDimensional Problems, Journal of Applied Physics, Vol. 89, pp. 1371-1376, 2001.

17. Lipton, R., Homogenization of the Product of Weakly Converging Sequences of Gradients, Technical Report No. MS-10-00-13, Mathematical Sciences, Worcester Polytechnic Institute, 2000.

18. Ekeland, I., and Temam, R., Convex Analysis and Variational Problems, NorthHolland, Amsterdam, Holland, 1976.

19. Kohn, R. V., And Strang, G., Optimal Design and Relaxation of Variational Problems, Communications on Pure and Applied Mathematics, Vol. 34, Part 1, pp. 113-137, Vol. 34, Part 2, pp. 139-182, Part 3, pp. 357-377, 1986. 
20. Lipton, R., And Velo, A. P., Optimal Design of Gradient Fields with Applications to Electrostatics, Proceedings of the College de France Seminar, Edited by D. Cioranescu and J. L. Lions, Pittman Research Notes in Mathematics, Pittman, London, England (to appear).

21. Grabovsky, Y., Optimal Design Problems for Two-Phase Conducting Composites with Weakly Discontinuous Objective Functionals, Advances in Applied Mathematics (to appear).

22. Pedregal, P., Constrained quasiconvexity and structural optimization. Archive for Rational Mechanics and Analysis, Vol. 154, pp. 325-342, 2000.

23. Pedregal, P., Fully Explicit Quasiconvexification of the Mean-Square Deviation of the Gradient of the State in Optimal Design, Electronic Research Announcments of the American Mathematical Society, Vol. 7, pp. 72-78, 2001.

24. Tartar, L., Remarks on Optimal Design Problems, Calculus of Variations, Homogenization and Continuum Mechanics, Edited by G. Buttazzo, G. Bouchitte, and P. Suquet, World Scientific, Singapore, pp. 279-296, 1994.

25. Raitums, U., Optimal Control Problems for Elliptic Equations: Mathematical Questions, Zinatne, Riga, Latvia, 1989. 